\title{
SEMBLANZA
}

\section{Efraín Montesinos: reparando corazones}

\author{
Efrain Montesinos: mending hearts
}

\author{
José Pacheco ${ }^{1}$ \\ ${ }^{1}$ Director de la revista Anales de la Facultad de Medicina, Universidad Nacional Mayor de San Marcos. Lima, Perú.
}

\section{Resumen}

La presente es una semblanza del cirujano cardiovascular Dr. Efraín Montesinos Mosqueira, Profesor de la Facultad de Medicina de San Fernando, de la Universidad Nacional Mayor de San Marcos quien, proveniente de una agreste pero hermosa serranía, fue formado para afrontar una de las más delicadas y eficientes cirugías, la cardiovascular y torácica. Este personaje no solo destacó en dicha cirugía en Ohio, EE UU, sino que luego la puso al servicio de los peruanos más necesitados económicamente, creando un programa (ahora servicio) de cirugía cardiovascular y torácica, en el Hospital Dos de Mayo, en donde dio atención gratuita a adultos y niños, a costa de muchos sacrificios, desvelos y frustraciones. Además, fue gestor -conjuntamente con la asociación médica de peruanos radicados en $\mathrm{EE}$ UU (PAMS) - de 70 misiones médicas, en 14 departamentos del Perú, que atendieron pacientes en diversas especialidades médicas y quirúrgicas y trajeron equipos y materiales, que luego quedaron en el país. Se hace este homenaje a un año de su fallecimiento.

Palabras clave: Montesinos, Efraín; cirugía torácica; docente médico; enfermedades cardiovasculares.

\begin{abstract}
This is a biographical sketch of Efrain Montesinos-Mosqueira, Professor of San Fernando Faculty of Medicine, San Marcos National University, whose infancy in a rough but beautiful mountainous region shaped him to face one of the most delicate but efficient surgeries, the cardiovascular and thoracic surgery. He did not only stood out in this specialty in Ohio, USA, and Peru, but he offered it to the most needed Peruvians, creating a program (now service) of cardiovascular and thoracic surgery, at Dos de Mayo Hospital, Lima, Peru, where he attended free of charge adults and children, despite sacrifices, pains and frustrations. He also conducted, along with de Peruvian American Medical Society (PAMS), 70 medical missions in 14 Peruvian departments that attended patients in diverse medical and surgical specialties and brought equipments and materials that remained in the country. We pay this homage commemorating one year from his departure.
\end{abstract}

Key words: Montesinos, Efrain; thoracic surgery; faculty, medical; cardiovascular diseases.
Un hombre valioso es aquél imprescindible para tareas gigantes, como las que realizó un Profesor de nuestra Facultad, el Dr. Efraín Montesinos Mosqueira, quien nunca pidió créditos, sino hizo el bien, gratuitamente, y a costa de muchos sacrificios, desvelos y frustraciones. Figura 1.

Efraín Montesinos nació el 17 de abril de 1937, en la Hacienda Tipín, Provincia de Grau, Apurímac, Perú, región de accidentada geografía, entre inmensos cerros y abismos profundos. En la región de Andahuaylas tuvieron su asiento los legendarios Chancas, conjunto de tribus que por su belicosidad han pasado a la historia como uno de los pueblos más aguerridos y rebeldes del Perú antiguo ${ }^{(1)}$. Ahí nacieron personajes como Micaela Bastidas, quien junto a su esposo José Gabriel Condorcanqui, Túpac Amaru II y Mateo Pumacahua, enfrentó la tortura del opresor español y dio su vida por la libertad.

Cuando Efraín aún no había nacido, perdió a su padre, destacado Capitán del Ejército, estando desde entonces su edu- cación a cargo de su madre y hermanos mayores. Como era costumbre en muchos lugares del Perú de la época, nuestros antepasados tenían como devoción el arte de curar a la gente. Así, su madre conoció las propiedades curativas de las plantas naturales y las empleaba para ayudar a sanar a su comunidad, influencia materna en la futura decisión de Efraín Montesinos de brindar salud a las personas.

Estos planos agrestes, de aires puros y cielo azul diáfano, de caminos entrecortados e interminables, formaron el carácter indomable de Efraín. Este futuro cirujano cardiovascular realizó sus estudios de primaria en la única Escuela Fiscal del lugar, que distaba $10 \mathrm{~km}$ de su casa, unas dos horas de distancia, que caminaba diariamente con gran entusiasmo. Seguramente, en esas ocasiones saboreaba la huatía, preparada desde épocas ancestrales con papas cocidas en hornos regionales, o papas y huevos con uchullachua (ají con queso fresco y huacatay), panes rejillas, tarapacos, paltos y roscas ${ }^{(2)}$. Estas caminatas también formaron su porte atlético y su dedicación a los deportes que mantendría en su vida y su perseverancia para llegar a destinos. Como aquella vez, mucho más adelante, en las punas de Bolivia, cuando en un viaje de promoción con sus compañeros de estudios de medicina, el bus quedó varado, y además del frío intenso, los muchachos no tenían alimentos ni agua. Efraín se puso su chompa y salió a cruzar montañas y valles, hasta encontrar comestibles y ayuda, con lo que salvó a sus entrañables amigos.

Su afán de superación se muestra en dos aspectos de su niñez, cuando adelantó materias de años superiores en la escuela, de manera de completar su ciclo de tres años en solo dos años. Y, cuando se trasladó a una escuela de Cusco, no queriéndolo el Director matricular en el cuarto grado -pues venía de un pueblo con una 'mala' escuela-, él demostró que sí se podía, al aprobar exitosamente el año, a pesar de los malos presagios del Director.

Los estudios secundarios los realizó en la Unidad Escolar Bartolomé He- 
rrera, en Lima, donde se distinguió por su aplicación a los estudios. Quedó subcampéon nacional en el concurso 'El que estudia triunfa', representando a su colegio. Se dice que siempre recordaba aquella pregunta que tuvo una respuesta ganadora '¿Dónde se firmó el tratado de fin de guerra de Perú y Ecuador de 1941? Su respuesta Mapaysingue, Mapaysingue, la repetía y repetía ${ }^{(3)}$, como repetiría siempre sus convicciones sobre aspectos médicos y quirúrgicos, sobre dar de sí mismo y sobre la necesidad del cuidado del paciente sin recursos.

Como deportista, obtuvo preseas doradas para su colegio, al obtener el Campeonato Interescolar Nacional de Atletismo, en la modalidad de Postas Chasqui. En el último año de secundaria, fue elegido Brigadier General del colegio, debido a sus méritos académicos, deportivos y su don de mando, obteniendo para su colegio el Gallardete Nacional en el Desfile de Fiestas Patrias. También, ese año consiguió el campeonato nacional de ciclismo, en la categoría novel, representando al Club Lobato de Lince.

Realizó su Educación Pre-Médica en la Universidad Nacional San Antonio Abad del Cusco, entre 1955 y 1956, y luego sus Estudios de Medicina en San Fernando, de la UNMSM, entre 1957 y 1962, recibiéndose de Médico Cirujano en noviembre de 1963. Como anécdota, siendo cachimbo le encontraron un soplo cardiaco y le prohibieron hacer deportes. A los tres años, le señalaron que dicho soplo era fisiológico y reasumió su vida deportiva, que mantuvo siempre. Se vislumbraba su deseo de conocer más sobre la fisiología de aquel órgano tan maravilloso, el corazón. Sus compañeros de la Promoción Héctor Colichón lo recordamos como el muchacho trabajador, amable, consejero, travieso e infatigable, con quien compartimos en el quinto año de medicina la creación del Club de Cirugía Experimental, comprando nuestros instrumentos quirúrgicos, estableciendo reglamentos de trabajo asistencial, obteniendo el permiso de las autoridades del Hospital Dos de Mayo para realizar las intervenciones, con anestesia, cuidados pre y postoperatorios, antibióticos, guardias, visitas médicas y todo lo necesario para que nuestros 'pacientes' salieran

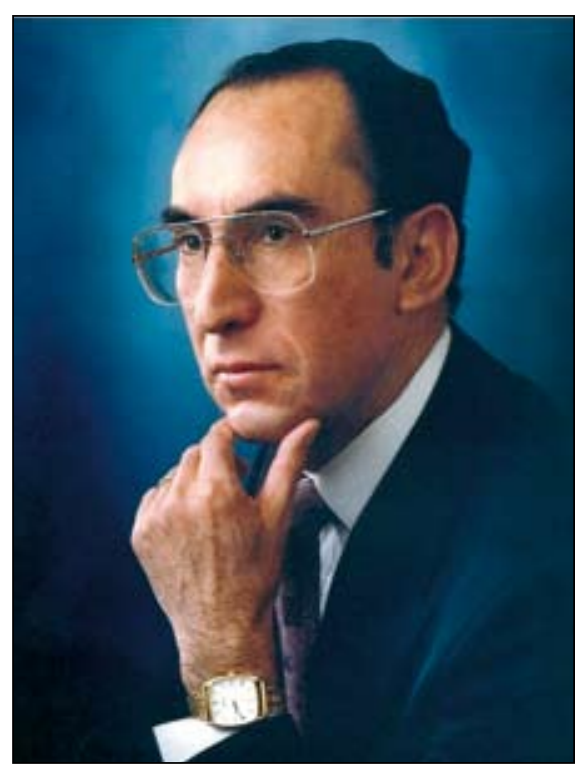

Figura 1. Efraín Montesinos. 17 abril 1937 - 28 diciembre 2007.

en las mejores condiciones a una larga vida.

Efraín Montesinos se ganaba su sustento como interventor de espectáculos en la Caja de Depósitos, lo cual permitió a algunos de sus compañeros sanfernandinos ingresar al cine gratis. Y también recordamos la crisis de $1961^{(4,5)}$, cuando un gran grupo de nuestros profesores dejaron la Facultad de Medicina de San Fernando y crearon su propia Universidad. Luego de tres meses de desconcierto, otros dignos profesores reactivaron la vida de la Facultad y Efraín se preocupó para que, quienes habíamos viajado a España, regresáramos a nuestra Facultad a retomar nuestros estudios. Efraín finalizó sus estudios entre los 10 mejores alumnos.

Hizo su Internado en el Hospital Obrero (1962 a 1963) y una Residencia en Cirugía de Tórax y Cardiovascular en el mismo hospital (1963 a 1964), la segunda residencia médica que fuera creada por la Facultad. Paralelamente, apoyó gratuitamente en la Posta Médica de la Ciudad de Papel del Padre Frisancho. Conformó el equipo que realizó la primera intervención cardiaca con circulación extracorpórea, en Lima, conjuntamente con los Drs. Smith y Peschiera, a un paciente con comunicación interauricular. En esta época, es que conoció a su compañera de toda la vida, María Cristina Puente de la Vega, con quien realizó no solo un víncu- lo de tierno esposo y de familia, sino que la vida de pareja se extendió a las tareas que vendrían fatigantes y a raudales en el futuro.

Posteriormente, se entrenó en Sao Paulo, Brasil, viajó a The Cleveland Clinic Foundation, con beca de la Fullbright Hays, entre 1972 y 1974, y realizó Cirugía General en el Medical College de Ohio, en Toledo, entre 1974 y 1977 . Obtuvo el grado de $\mathrm{PhD}$ en la Cleveland Clinic Foundation, en setiembre de 1969.

En Lima, recibió el grado de Doctor en Medicina, en San Fernando, UNMSM, en marzo de 1972 y, entre abril de 1967 y junio de 1972, fue cirujano de tórax y cardiovascular en el Hospital Obrero de Lima. En este lapso, realizó la primera revascularización miocárdica, con la técnica de Vine Berg (febrero 1970), el primer by-pass aorto coronario con safena (mayo 1970), resección de aneurisma del ventrículo izquierdo postinfarto de miocardio y revascularización miocárdica con conductos arteriales (mamaria interna), en $1972^{(3)}$.

No teniendo facilidades para practicar la cirugía especializada al ritmo que él deseaba, regresó a los EE UU y, de julio 1977 a marzo 1978, fue miembro junior del staff de la Cleveland Clinic Foundation y, entre abril 1978 y 1996, miembro del Cardiovascular and Thoracic Consultants, Inc., teniendo posteriormente privilegios como cirujano de cirugía torácica y cardiovascular en la práctica privada en los hospitales norteamericanos de la ciudad de Ohio The Toledo Hospital, Medical College Hospital, St. Luke Hospital, St. Vincent Hospital, Mercy Hospital. Encontramos un título de un artículo sobre Efraín en la revista Profile de la Cleveland Clinic, $1984{ }^{(6)}$, que dice 'A surgeon's skill: helping hearts that hurt', 'La destreza de un cirujano: ayudando a los corazones adoloridos'. "El Dr. Efraín Montesinos ha estado cientos de veces en esta sala de operaciones, plantado en el mismo lugar, sobre la misma mesa, remendando corazones. Pero, él no se apura, delibera, es minucioso. Con su vestimenta azul y lentes de magnificación especiales, él trabaja con suma precisión".

Tuvo certificaciones y licencias del Colegio Médico del Perú, Estado de 


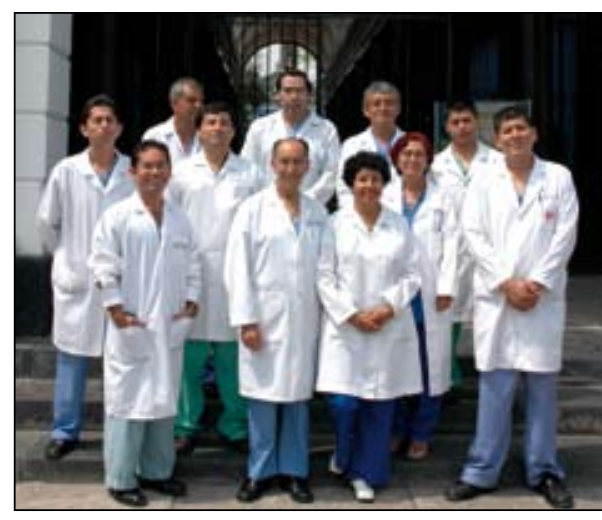

Figura 2. El Dr. Montesinos y personal médico del Programa de Cirugía de Tórax y Cardiovascular, Hospital Nacional Dos de Mayo

Ohio, The American Board of Surgery y The American Board of Thoracic Surgery. Fue declarado Profesor Emérito de la Universidad Ohio. Luego, vino la jubilación.

La labor de Montesinos podría haber concluido allí. Pero, no. Él tenía ideas magníficas y sumamente necesarias en mente. En el año 1994, inició una serie de Misiones Médicas en las regiones pobres del Perú ${ }^{(7)}$. Empezó en Cusco y siguió en Arequipa, Trujillo, Ayacucho, Iquitos, Lima, Huánuco, Chiclayo, Abancay, Huancavelica y otras ciudades del Perú. Su idea fue que los miembros de la Peruvian American Medical Society -PAMS- revirtieran en el Perú lo que obtuvieron con la educación médica, de preferencia en su propia provincia. No solo hacer donativos, sino venir personalmente a atender a pacientes de las regiones remotas del Perú, así como intercambiar experiencias con los profesionales de la localidad. Y trajo médicos, anestesiólogos, enfermeras y personal paramédico norteamericano, además de equipos y materiales, que luego queda-

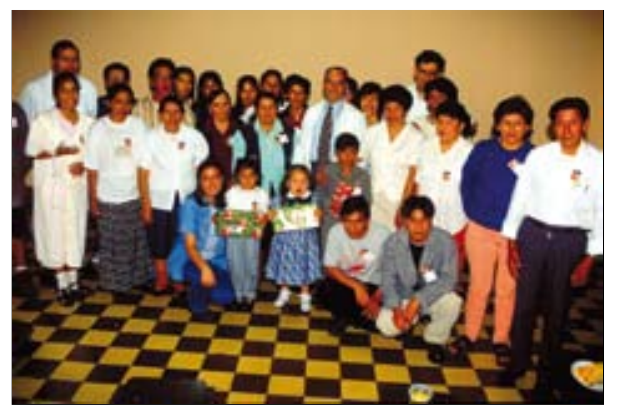

Figura 3. Pacientes y niños operados en el Hospital Nacional Dos de Mayo. ron en el Perú, en aquellas regiones distantes, venciendo todas las trabas burocráticas y físicas que involucra esta actividad misionera. Hasta junio de 2006 había realizado 70 Misiones Médicas en 14 departamentos del país, que atendieron 66057 pacientes en diversas especialidades médicas y operado a 6798 pacientes en la mayoría de especialidades quirúrgicas ${ }^{(7,8)}$. Actividad médico-quirúrgica que considero pionera y sin parangón.

Y, desde noviembre de 1998 hasta el 2007, fue Director del Programa de Cirugía de Tórax y Cardiovascular del Hospital Nacional Dos de Mayo, una misión médica permanente y humanitaria dedicada a la cirugía cardiotorácica, gratuita, en beneficio de los pobres, de la población indigente del Perú ${ }^{\left({ }^{9}\right)}$. Figuras 2 y 3.

Contra viento y marea, y a fuerza de coraje y paciencia, obtuvo los permisos para equipar totalmente salas quirúrgicas para este tipo de cirugía, consultorios externos, laboratorio. Conociendo nuestra epidemiología y la necesidad de la población peruana, principalmente la de escasos recursos, extendió el servicio a los niños con malformaciones cardiacas congénitas y equipó y preparó cuadros en anestesiología y hemodiálisis. Él y María obtenían los equipos y materiales quirúrgicos en EE UU -con el apoyo de la PAMS-, coordinaban los documentos, permisos y el transporte, lograban el fatigante desaduanaje, conseguían los camiones para transportar todo a su querido hospital, a su querido servicio y a sus queridos pacientes. Labor que, inicialmente, no fue entendida por sus colegas especialistas, algunos médicos del Hospital y algunos Profesores sanfernandinos. Sin embargo, ostenta el título de Profesor Honorario de Cirugía, de la Universidad Nacional de San Agustín de Arequipa, Distinción Humanitaria de la The Peruvian American Medical Society, Inc., el Tumi de Oro por el mejor desempeño médico en el Perú, de la Asociación Médica Peruana, y un Reconocimiento Especial del Departamento de Cirugía del Hospital Dos de Mayo, por la exitosa organización del Programa de Cirugía Cardiotorácica, para intervenciones humanitarias de los más pobres de los pobres, en el año 2004. Entre otras, su promoción médica 1962, Héctor Colichón Arbulú, realizó un actividad académica el 4 de diciembre de 2007, en el local del Colegio Médico del Perú, en el que Efraín Montesinos recibió sendas Medallas al Mérito Extraordinario de la Facultad de Medicina de San Fernando (en la que ejerció la docencia desde 1958) y del Consejo Nacional del Colegio Médico del Perú, Medalla del Rectorado de la Universidad Nacional Mayor de San Marcos y el reconocimiento de sus compañeros de promoción, de sus compañeros de labores del Hospital Dos de Mayo y de sus pacientes.

Efraín Montesinos publicó 34 trabajos de la especialidad en prestigiosas revistas internacionales y 42 abstracts, comunicaciones preliminares, discusiones de panel, pudiendo decirse que influyó en la evolución de la cirugía cardiaca a través de organizaciones importantes y fue un portavoz persuasivo de esta rama tan importante de la cirugía. Perteneció a numerosas sociedades médicas nacionales e internacionales -entre ellas Miembro Fundador de la Sociedad Mundial de Cirugía Pediátrica y Cardiaca Congénita-, ejerció la presidencia de varias instituciones, recordándose el impulso otorgado cuando dirigió la Peruvian American Medical Society.

Su servicio a la comunidad y/o membresías organizacionales incluye el ser Fundador y Miembro Vitalicio de Los Corazones Remendados de Cap Glass City, Director Médico en el Perú de Heartbeat International, Miembro del Museo de Arte de Toledo y del Ballet y Ópera de Toledo, Miembro del Rotary Club de Toledo, de El Rímac y del Club Sunrise de Lima y Director Médico de la Fundación Peruana Cardioinfantil, entre otros.

Somos testigos del amor que profesó a su familia, a su esposa María Cristina y sus hijos María Cecilia, Efraín, María Claudia, Susana María, de una ternura pocas veces vista, de amor cristiano, de amor humano, de entrega y servicio, el mismo que extendió a sus amigos y a sus pacientes, especialmente a aquellos a quienes el destino no proveyó con las condiciones sociales y económicas que 
les permitiera vivir adecuadamente. Así, continuó preocupándose de atender a los pacientes de su servicio cardiotorácico, a pesar de la penosa enfermedad que lo aquejaba y que puso fin a su brillante trayectoria, el 28 de diciembre de 2007. Su caminar desde la serranía, los abismos, ríos y caballos hasta la universidad, la carrera médica, los triunfos y decepciones, solo han hecho crecer a este hombre en la dimensión histórica peruana, hasta convertirlo en una leyenda, ejemplo para nosotros y para quienes nos siguen en la actividad profesional, en la docencia y en la vida. Que los médicos peruanos puedan seguir su vida ejemplar.

\section{REFERENCIAS BIBLIOGRÁFICAS}

1. Historia de Andahuaylas [monografía en la Internet]. Sunnyvale, CA: GeoCities [citado 28 de noviembre de 2007]. Disponible en: http://www. geocities.com/ozperu/historia.html
2. Departamento de Apurímac [monografía en la Internet]. Sunnyvale, CA: GeoCities [citado 28 de noviembre de 2007]. Disponible en: http://www. geocities.com/gil2000_pe/apurimac.html

3. Peralta J. Semblanza Dr. Efraín Montesinos. II Convención Nacional de Cirugía de Tórax y Cardiovascular "Efraín Montesinos Mosqueira". Lima, Perú. 19 marzo 2008.

4. Pacheco J. Anales de la Facultad de Medicina, Un recuento, 1918 a 2008, con ocasión del $90^{\circ}$ aniversario de la Revista. An Fac med. 2008;69 Supl 2:9-16.

5. Murillo JP, Franco G. San Fernando: ¿utopía o proyecto inconcluso?. An Fac med. 2008;69(2):130-9.

6. The Toledo Hospital. A surgeon's skill helping hearts that hurt. Profile. 1984;2(3):15-7.

7. Peruvian American Medical Society. Medical Missions Committee. Misiones Médicas al Perú. Marzo 1994-diciembre 2006 [monografía en la Internet]. Atlanta, Georgia: The Society; 2006 [citado 15 de setiembre de 2008]. Disponible en: http://www. pamsnational.org/files/PACIENTES\%20ATENDID OS\%20Y\%200PERADOS.doc

8. Peruvian American Medical Society. Efraín Montesinos y las misiones médicas. XXXV PAMS
Convention. Cajamarca, Perú, July 9-12, 2008.

9. Montesinos E, Peralta J, Rojas L, Adrianzén M, Zokolich J, Yaryhuamán AR. Cirugia valvular en el Hospital Dos De Mayo. Revista Angioplastía Perú [revista en la Internet]. Octubre 2002 [citado 15 de setiembre de 2008]. Disponible en: http://www. angioplastiaperu.com/revista/articulos02.htm

Manuscrito recibido el 10 de julio de 2008 y aceptado para publicación el 15 de agosto de 2008.

Correspondencia:

Dr. José Carlos Pacheco Romero

Director Revista Anales de la Facultad de Medicina, Universidad Nacional Mayor de San Marcos

Av. Grau 755. Lima 1, Perú

Correo-e: jpachecoperu@yahoo.com 